\title{
Mecanismos de autofosforilación y transfosforilación en sistemas de dos componentes bacterianos
}

\author{
Juan Luis Terán-Melo, Claudia Rodríguez-Rangel, \\ Dimitris Georgellis y Adrián F. Álvarez* \\ Departamento de Genética Molecular, Instituto de Fisiología Celular, Universidad Nacional Autónoma de \\ México. Ciudad Universitaria, Ciudad de México, 04510, México. *E-mail: aalvarez@ifc.unam.mx
}

\begin{abstract}
RESUMEN
Los Sistemas de señalización de Dos Componentes (SDCs) permiten a las bacterias detectar estímulos ambientales y responder a estos de manera adaptativa. Estos sistemas de transducción de señales se basan en la autofosforilación y transferencia de grupos fosforilo entre residuos de histidina y aspartato de una proteína cinasa sensora y un regulador de respuesta. Cuando la cinasa sensora percibe un estímulo específico se autofosforila, en una reacción que puede ser inter o intramolecular, y transfosforila a su regulador de respuesta cognado, que actúa generalmente como regulador transcripcional para ejercer una respuesta fisiológica. Con frecuencia, en ausencia de estímulo, la misma cinasa sensora se encarga de desfosforilar a su regulador de respuesta cognado. Además, algunas cinasas sensoras tienen módulos adicionales que participan en un fosforelevo que termina en la fosforilación del regulador de respuesta, y en un fosforelevo reverso que permite su desfosforilación. Al igual que en la autofosforilación, las trasferencias de grupos fosforilo implicadas en el fosforelevo y el fosforelevo reverso pueden ser inter o intramoleculares. En esta revisión exponemos algunas de las más importantes características de los SDCs bacterianos, poniendo especial énfasis en los procesos de autofosforilación y fosfotransferencia.
\end{abstract}

Palabras Clave: cinasa sensora, autofosforilación, transfosforilación, intermolecular, intramolecular.

Autophosphorylation and transphosphorylation mechanisms in bacterial two component systems

\begin{abstract}
Two-component signaling circuits (TCS) allow bacteria to detect environmental cues and to produce adaptive responses. These signaling systems are based on autophosphorylation and phosphoryl-group transfers between histidine and aspartate containing sensor kinase and response regulator proteins. Upon reception of a specific stimulus the sensor kinase protein autophosphorylates, by either an inter- or intra-molecular reaction, and transphosphorylates its cognate response regulator, which, typically, acts as a transcriptional regulator, thereby triggering physiological responses. Frequently, in the absence of the stimulus, the sensor kinase proteins are responsible for the dephosphorylation of their cognate response regulators. Furthermore, a group of sensor kinases have additional functional domains that are involved in a forward phosphorelay for signal transmission and in a reverse phosphorelay for signal decay. As is the case of the autophosphorylation reaction, the phosphoryl-group transfers involved in the forward and reverse phosphorelay can occur either intra- or inter-molecularly. In this review, we highlight some important features of bacterial TCS, with special emphasis on the autophosphorylation and phosphoryl-group transfer events.
\end{abstract}

Key words: sensor kinase, autophosphorylation, transphosphorylation, intermolecular, intramolecular.

Nota: Artículo recibido el 04 de septiembre del 2018 y aceptado el 07 de febrero del 2019. 


\section{INTRODUCCIÓN}

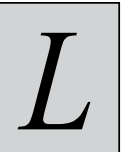

os organismos necesitan comunicarse y recibir información del medio que los rodea, para así adaptarse rápidamente a las condiciones imperantes. A nivel celular, esta comunicación se da a través de sistemas de transducción de señales (aunque no todas las respuestas adaptativas requieren de transducción de señales), que funcionan como vías procesadoras de información intracelular, acoplando cambios ambientales con cambios fisiológicos que resultan en respuestas adaptativas. A pesar de la gran diversidad de estímulos y respuestas, las células utilizan sólo un pequeño número de estrategias moleculares para la transducción de señales, siendo la fosforilación de proteínas una de las principales. En organismos eucariontes, las cascadas de señalización involucran proteín-cinasas que pueden autofosforilarse o fosforilar a otras proteínas en residuos de serina (Ser), treonina (Thr) o tirosina (Tyr). Sin embargo, en los sistemas de señalización bacteriano, predomina un esquema de fosforilación diferente, en donde están involucrados residuos de histidina(His) y aspartato(Asp). Estas vías de señalización están conformadas frecuentemente por dos proteínas: una cinasa sensora (CS) y un regulador de respuesta (RR), por lo que son conocidas como Sistemas de señalización de Dos Componentes (SDCs). Cada uno de estos sistemas está altamente especializado, de manera que detectan una o unas pocas señales moleculares, producto de una condición ambiental, y permiten una respuesta específica que es, en la mayoría de los casos, producida por una expresión génica diferencial. Con pocas excepciones, todas las bacterias tienen múltiples SDCs (Galperin, 2005; Ulrich \& Zhulin, 2010; Wuichet, Cantwell, \& Zhulin, 2010), necesarios para su adaptación a cambios en condiciones ambientales, como osmolaridad, balance redox, $\mathrm{pH}$, temperatura, disponibilidad de nutrientes, etc. (Aguilar, Hernández-Arriaga, Cybulski, Erazo, \& de Mendoza, 2001; Eguchi \& Utsumi, 2014; Forst \& Roberts, 1994; Iuchi \& Lin, 1993; Nohno, Noji, Taniguchi, $\&$ Saito, 1989). Además, tienen un papel relevante en la regulación de diversos procesos celulares como esporulación, resistencia a antibióticos, motilidad y patogénesis (Bourret, Hess, Borkovich, Pakula, \& Simon, 1989; Hutchings, Hong, \& Buttner, 2006; Jiang, Shao, Perego, \& Hoch, 2000).

Cabe destacar que los SDCs no son exclusivos de las bacterias, ya que también se encuentran presentes en la mitad de los genomas secuenciados de arqueas (Ashby, 2006), y están presentes en levaduras, hongos, amebas sociales y plantas (Catlett, Yoder, \& Turgeon, 2003; Koretke, Lupas, Warren, Rosenberg, \& Brown, 2000; Santos \& Shiozaki, 2001; Schaller, Shiu, \& Armitage, 2011; Thomason \& Kay, 2000; Wolanin, Thomason, \& Stock, 2002).

\section{FunCIONAMIENTO DE LOS SDCS BACTERIANOS}

En un SDC bacteriano típico, la CS es una proteína membranal y el RR es una proteína soluble que generalmente actúa como regulador transcripcional. La percepción de una señal específica por la CS permite su autofosforilación, dependiente de ATP, en un residuo de His que se encuentra en el dominio de la proteína que se denomina transmisor (DT) (Figura 1). Inmediatamente, el grupo fosforilo $(\sim P)$ es transferido a un Asp en el RR, en un dominio que se conoce como receptor (DR) (Figura 1a). El RR fosforilado (RR-P) media la respuesta celular mediante la regulación de la expresión de genes a nivel transcripcional. Por otra parte, cuando la señal específica está ausente, el RR-P pierde el $\sim \mathrm{P}$, inactivándose como regulador transcripcional (Figura 1a). Por lo general, la desfosforilación del RR es acelerada por la misma CS cognada que, en ausencia de la señal, adquiere una actividad fosfatasa específica para su RR fosforilado (Kenney, 2010; Stock, Robinson, \& Goudreau, 2000). No obstante, en algunos SDCs bacterianos, y en la mayoría de los que se encuentran en eucariontes, la CS cuenta con dominios proteicos adicionales que participan en la transducción de la señal. En estas CS, denominadas híbridas, además del DT, existe un dominio receptor adicional (DR), con un Asp conservado, y un dominio de fosfotransferencia (HPt), con una His conservada (Figura 1b). Cuando estos sistemas son activados por una señal específica, la CS híbrida se autofosforila en el dominio transmisor, y posteriormente transfiere el $\sim$ P secuencialmente al Asp del DR y a la His del HPt dentro de la CS, para finalmente fosforilar al Asp del RR (Figura 1b) (Burbulys, Trach, \& Hoch, 1991; Georgellis, Lynch, \& Lin, 1997; Takeda, Fujisawa, Matsubara, Aiba, \& Mizuno, 2001; Uhl \& Miller, 1996b; Williams \& Whitworth, 2010). A este proceso se le conoce como fosforelevo. Nuevamente, cuando la señal está ausente, el RR-P se desfosforila, reacción que es catalizada por la misma CS, pero en este caso mediante un fosforelevo reverso, que involucra la transferencia del $\sim \mathrm{P}$ del RR a la His del HPt, luego al residuo de Asp del DR de la CS híbrida, y finalmente la liberación de fosfato inorgánico (Figura 1b) (Georgellis, Kwon, De Wulf, \& Lin, 1998; Peña-Sandoval, Kwon, \& Georgellis, 2005; Uhl \& Miller, 1996a).

\section{LOS SDCS SON SISTEMAS MODULARES}

Como se mencionó previamente, la transducción de la señal a través de un SDC involucra la transferencia de $\sim \mathrm{P}$ entre dominios proteicos. Un aspecto interesante del funcionamiento de los SDCs es que cada dominio que los compone opera de manera autónoma e independiente. En algunos sistemas bacterianos, y en la mayoría de los de células eucariotas, la CS híbrida está compuesta sólo por los dominios DT y DR, mientras que el dominio HPt forma parte de una proteína separada. La naturaleza modular de los SDCs se hace evidente en CSs híbridas como ArcB de Escherichia coli, en donde se comprobó que el fosforelevo ocurre de igual manera si los tres dominios (DT, DR y HPt) están formando parte de la misma proteína (ArcB), o si estos se expresan intencionalmente en péptidos separados (Georgellis, Lynch, \& Lin, 1997). Esto sugiere que los diferentes dominios catalíticos que componen 
A)

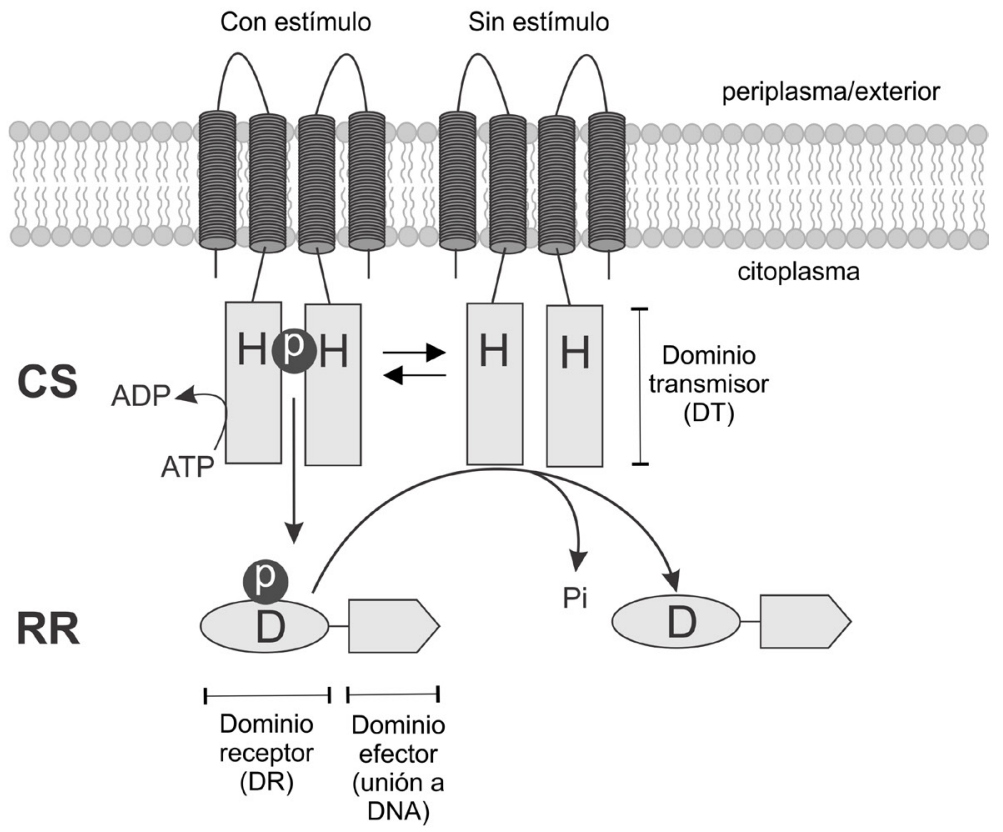

B)

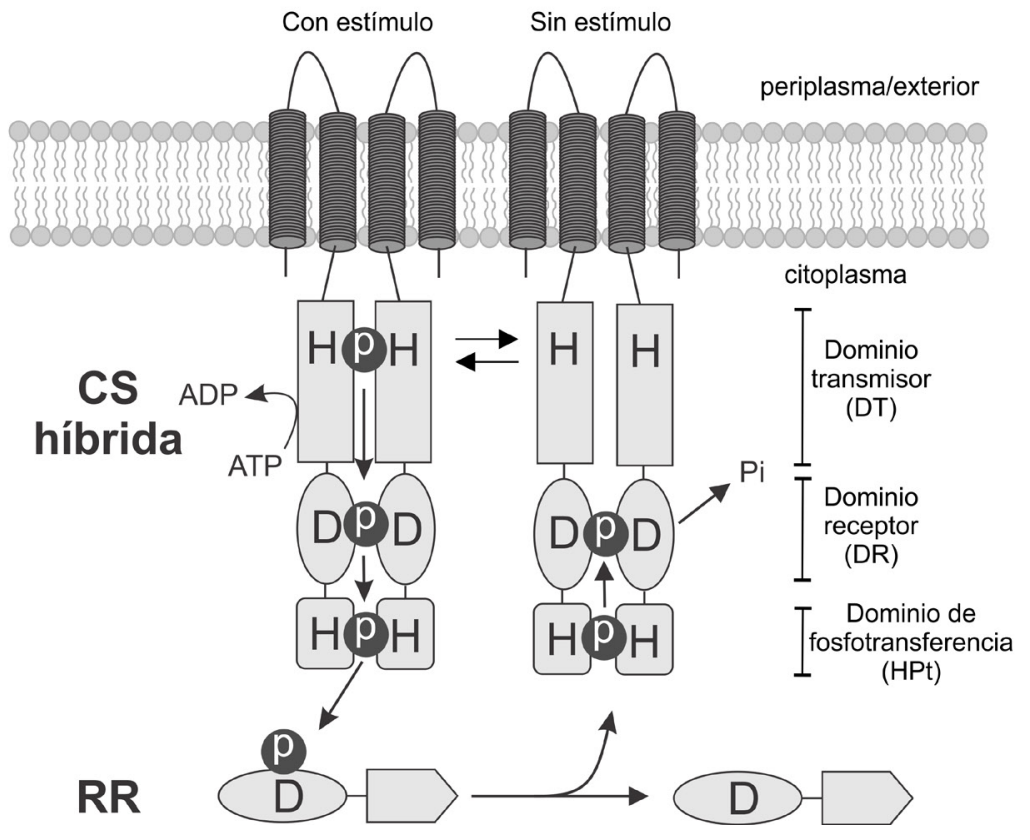

Figura 1. Representación esquemática de los sistemas de dos componentes bacterianos y su funcionamiento. A) SDC canónico con una CS membranal y un RR citosólico. Se muestra el dominio transmisor (DT) de la CS, con un residuo de His (H) que se fosforila en condiciones estimulantes, y el dominio receptor (DR) del RR, con un residuo de Asp (D) conservado que recibe el grupo fosforilo de la CS. En condiciones no estimulantes, la CS funciona como fosfatasa y cataliza la desfosforilación del RR-P. B) SDC con una CS híbrida, que contiene el DT, un DR con un Asp (D) conservado, y un dominio HPt, con una His (H) también conservada. La fosforilación del RR se concreta mediante un fosforelevo. Cuando la señal está ausente, la CS híbrida cataliza la desfosforilación del RR-P mediante un fosforelevo reverso, que termina en la liberación de fosfato inorgánico (Pi). Fuente: elaboración propia. 
un SDC han evolucionado de forma que cada uno puede operar de manera autónoma, interactuando con los demás módulos específicos aunque no exista un enlace covalente manteniéndolos en cercanía (Georgellis, Kwon, De Wulf, \& Lin, 1998; Georgellis, Lynch, \& Lin, 1997).

\section{DOMINIO TRANSMISOR： DIMERIZACIÓN Y AUTOFOSFORILACIÓN}

El dominio transmisor es la parte catalítica de la CS que permite la autofosforilación en un residuo de His conservado. Por función y estructura este módulo proteico está compuesto por dos subdominios: el llamado dominio de dimerización e histidín-fosfo transferencia (DHp), y el dominio catalítico y de unión a ATP (CA) (Figura 2). El primero está formado por dos $\alpha$-hélices que están unidas por un bucle y en la primera de las hélices se encuentra la His que es fosforilada. El segundo es un dominio globular conformado por 5 láminas $\beta$-plegadas flanqueadas por 3 hélices, que contienen el sitio de unión a ATP. Como su nombre lo indica, el dominio DHp permite la formación de dímeros estables y es el responsable de que la gran mayoría de las CSs se encuentren y funcionen al menos como homodímeros (Dutta, Qin, \& Inouye, 1999; Levit, Liu, Surette, \& Stock, 1996; Tanaka et al., 1998; West \& Stock, 2001). Teniendo en cuenta la naturaleza dimérica de las CSs, existen dos modos posibles de autofosforilación: intramolecular (en cis), en donde el ATP unido al dominio CA de un monómero fosforila a la His del dominio DHp del mismo monómero; o intermolecular (en trans), en donde el ATP se une al dominio CA de un monómero para fosforilar a la His del otro monómero. Trabajos pioneros realizados a principios de los años 90 se propusieron averiguar cuál era el modo de autofosforilación en algunas CSs. Para ello, usaron versiones mutantes puntuales de la CS en uno o varios aminoácidos esenciales para la unión del ATP. Como era de esperar, estas CSs eran inactivas tanto in vivo como in vitro, ya que no eran capaces de autofosforilarse. Luego, intentaron complementar funcionalmente a esta mutante, con otra mutante puntual de la misma CS, pero ahora con un reemplazo de la His conservada en el dominio DHp (Figura 2a). En todos los casos, se encontró que los heterodímeros de una CS, con un monómero mutante en la His y el otro mutado en el dominio de unión a ATP (dominio CA), eran capaces de autofosforilarse. Así, se concluyó que las CSs EnvZ, NtrB y CheA se autofosforilan mediante una reacción intermolecular (en trans) (Ninfa, Atkinson, Kamberov, \& Ninfa, 1993; Swanson, Bourret, \& Simon, 1993; Yang \& Inouye, 1991). Posteriormente, se determinó la naturaleza intermolecular de la autofosforilación de otras CSs (Tabla I). Con estos hallazgos, se estableció la noción de que todas las CSs tienen el mismo modo de autofosforilación; además esto le da sentido al hecho de que la gran mayoría de las CSs operan como homodímeros. Sin embargo, diferentes grupos de investigación encontraron recientemente que existe autofosforilación intramolecular en CSs que, a pesar de ello, funcionan también como homodímeros. Este es el caso de las CSs HK853 de Thermotoga maritima, PhoR de Staphylococcus aureus (Casino, Rubio, \& Marina, 2009) y ArcB de E. coli (Kinoshita-Kikuta, Kinoshita, Eguchi, \& Koike, 2016; Peña-Sandoval \& Georgellis, 2010). Por lo tanto, parece que ambos modos de autofosforilación ocurren en cinasas de histidina bacterianas.

\section{DETERMINANTES DEL MODO DE AUTOFOSFORILACIÓN, CIS O TRANS.}

Diversos estudios demostraron que no todas las CSs se ajustan al paradigma de una autofosforilación en trans, sino que también hay algunas que lo hacen en cis (Tabla I). Por lo tanto, surge la siguiente cuestión: ¿qué determina que una CS se autofosforile en cis o en trans? Mediante la comparación de las estructuras cristalográficas de las regiones citosólicas de EnvZ (trans) y HK853 (cis), el grupo del Dr. Laub identificó una diferencia en la direccionalidad de las $4 \alpha$-hélices que se forman cuando los correspondientes dominios DHp dimerizan (Ashenberg, Keating, \& Laub, 2013). Esta direccionalidad está dada por un bucle o conector entre las dos $\alpha$-hélices que conforman el dominio DHp (Figura 2b). La secuencia primaria de aminoácidos y la longitud de este conector no están muy conservadas en las diferentes CSs; sin embargo, mediante la construcción y caracterización de quimeras de EnvZ (se fosforila en trans) con el conector de la CS PhoR (se fosforila en $c i s$ ), se determinó que la direccionalidad de las $\alpha$-hélices, determinada por esta pequeña secuencia, es suficiente para determinar el modo de autofosforilación. De esta manera se propuso un modelo que plantea que el posicionamiento de la hélice $\alpha-2$ hacia la derecha de $\alpha-1$, determinada por el conector, favorece una autofosforilación en trans, mientras que si la hélice $\alpha-2$ se orienta hacia la izquierda de la hélice $\alpha-1$, la fosforilación ocurre en cis (Figura 2b) (Ashenberg, Keating, \& Laub, 2013). Sin embargo, la cinasa DesK parece ser la excepción a la regla para este modelo. En DesK, el conector está orientado hacia la izquierda pero los estudios bioquímicos indican que la autofosforilación en esta CS ocurre en trans (Trajtenberg, Graña, Ruétalo, Botti, \& Buschiazzo, 2010). Por lo tanto, es posible que otros factores estructurales, que deben ser investigados de manera particular, influyan también en el modo de autofosforilación de una CS.

\section{TRANSFOSFORILACIÓN DEL RR Y FOSFORELEVO EN CSS HÍBRIDAS}

Una vez que un SDC es activado por una señal específica y la CS se autofosforila, la información es transmitida al aparato transcripcional mediante la transfosforilación del RR cognado. Este paso involucra una sola reacción de transferencia del grupo $\sim \mathrm{P}$ si la $\mathrm{CS}$ es canónica. Varios mecanismos aseguran la especificidad de esta reacción, reduciendo la posibilidad de que una CS fosforile a un RR que no sea su cognado (Álvarez, Barba-Ostria, Silva-Jiménez, \& Georgellis, 2016). Sin embargo, si el sistema está formado por una CS híbrida, la fosforilación del RR está precedida 
A)

Ensayo típico de complementación intermolecular

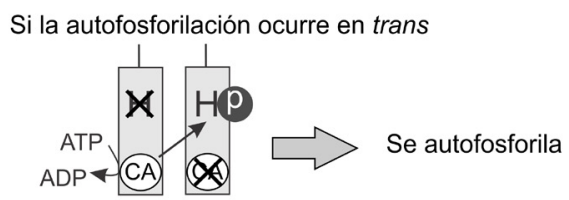

Si la autofosforilación ocurre en cis

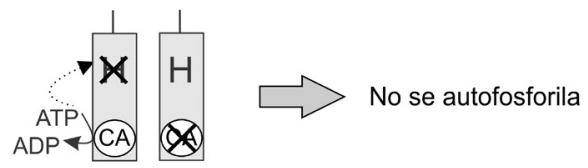

B)

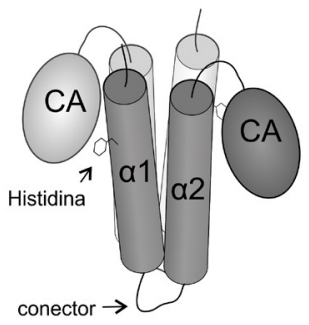

Autofosforilación en trans

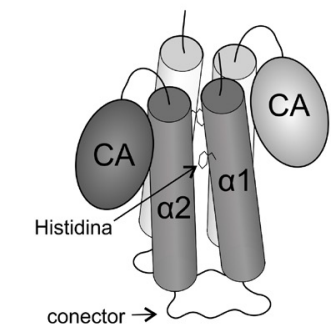

Autofosforilación en cis

Figura 2. Estructura y análisis del dominio transmisor. A) Esquema de un experimento típico de complementación intermolecular para discernir entre una autofosforilación intramolecular o intermolecular. La mezcla o co-expresión de proteínas CSs mutantes en la His conservada y en el domino de unión a ATP (dominio CA) puede resultar en la fosforilación de la CS si la autofosforilación ocurre en trans, o en la formación de dímeros inactivos si la fosforilación ocurre en cis. La mutación puntual está representada por una " $X$ ". B) Representación esquemática de los determinantes estructurales para la autofosforilación en trans o en cis. Cada monómero de dominio transmisor, compuesto por dos $\alpha$-hélices ( $\alpha 1$ y $\alpha 2)$, que conforman el subdominio DHp, y el subdomino de unión a ATP (CA), se encuentra representado con un tono de gris diferente. La naturaleza del conector entre la $\alpha 1$ y $\alpha 2$ determina la cercanía de la His conservada en la $\alpha 1$ con el dominio CA del mismo o del otro monómero, resultando en una autofosforilación en cis o en trans, respectivamente. Fuente: elaboración propia.

por dos reacciones de transferencia del grupo $\sim \mathrm{P}$ entre dominios de la CS (Georgellis, Lynch, \& Lin, 1997; Jourlin, Ansaldi, \& Mejean, 1997; Kwon, Georgellis, \& Lin, 2000; Uhl \& Miller, 1996b). Nuevamente, considerando que las CSs híbridas operan también como homodímeros, existen dos posibilidades en cada paso de fosfotransferencia del fosforelevo: inter o intramolecular. En contraste con lo que se pensaba inicialmente del modo de autofosforilación de las
CSs (se creía que era siempre intermolecular), desde un inicio se observó que no había patrones establecidos, y que el modo de fosfotransferencia dependía de la CS híbrida de la que se tratase, ya que a fines de los 90 se obtuvieron evidencias de que las transferencias del grupo $\sim \mathrm{P}$ del DT al DR y del DR al dominio HPt de la CS híbrida BvgS, ocurren en cis y trans, respectivamente (Uhl \& Miller, 1996a); mientras que en la CS híbrida TorS se observó que ambos pasos ocurren en trans (Jourlin, Ansaldi, \& Mejean, 1997). Además, recientemente se caracterizó el fosforelevo de otras CSs híbridas encontrando diferentes modos de fosfotransferencia (Kinoshita-Kikuta, Kinoshita, Eguchi, \& Koike, 2016; Terán-Melo et al., 2018), reforzando la idea de que cada sistema evolucionó adoptando características estructurales particulares que inciden sobre el modo de fosfotransferencia. En cualquier caso, se puede suponer que el patrón de transferencia del grupo $\sim \mathrm{P}$ entre los dominios catalíticos de una CS híbrida, es producto del acomodamiento de dichos módulos en la estructura tridimensional del homodímero, teniendo en cuenta que para que ocurra la transferencia debe haber una interacción o contacto cercano entre los dominios que intervienen. En contraste con lo que ocurre en la autofosforilación, no se tienen evidencias de que regiones específicas de la proteína, como las que conectan dos dominios catalíticos, determinen el modo de transferencia. La elucidación de la estructura cristalográfica de la porción citosólica de CSs híbridas podría ayudar a entender el por qué una CS híbrida presenta tal o cual modo de transfosforilación. Por el contrario, el conocer la naturaleza de las transferencias de grupos $\sim \mathrm{P}$ entre dominios de una CS híbrida, puede dar información sobre las características estructurales de esa cinasa. Más adelante en esta revisión se profundizará sobre esta idea con los hallazgos recientes del fosforelevo en la CS híbrida ArcB.

\section{DESFOSFORILACIÓN DEL RR-P}

Cuando la señal específica está presente, una CS se autofosforila y se inicia una serie de eventos que terminan en la regulación de la expresión génica por el RR cognado fosforilado. Cuando las condiciones ambientales cambian y la señal está ausente, el RR-P debe ser rápidamente desfosforilado. La reacción de desfosforilación del RR es un evento importante en las respuestas adaptativas, debido a que es perjudicial para las células seguir teniendo una repuesta a una condición ambiental que ya no existe. En efecto, muchas CSs son bifuncionales, es decir, además de participar en la fosforilación de su RR cognado (actividad cinasa) son capaces de desfosforilar específicamente a al RR-P (actividad fosfatasa) (Gao \& Stock, 2009). Esta actividad ha sido observada tanto en CSs canónicas como híbridas. En las primeras, el dominio transmisor participa en la actividad fosfatasa. Sin embargo, se ha descartado que esta actividad se realice mediante una fosfotransferencia reversa, ya que la His conservada en CSs como EnvZ no es esencial para la actividad fosfatasa. Por el contrario, se ha sugerido que la actividad fosfatasa de las 


\begin{tabular}{|c|c|c|c|c|c|c|}
\hline $\begin{array}{l}\text { Cinasa } \\
\text { sensora }\end{array}$ & Organismo & $\begin{array}{l}\text { Autofos- } \\
\text { forilación }\end{array}$ & $\begin{array}{l}\text { Tipo de } \\
\text { sensor }\end{array}$ & Genes / procesos regulados & $\begin{array}{l}\text { Responde a / } \\
\text { señal }\end{array}$ & Referencia \\
\hline EnvZ & E. coli & trans & Canónico & $\begin{array}{l}\text { ompC, ompF, fadL, tppB, } \\
\text { regulación de la biosíntesis } \\
\text { de flagelo y formación de } \\
\text { biopelícula. }\end{array}$ & $\begin{array}{l}\text { Cambios en la } \\
\text { osmolaridad del } \\
\text { medio }\end{array}$ & (Yang \& Inouye, 1991) \\
\hline NtrB & E. coli & trans & Canónico & $\begin{array}{l}\text { Glutamina sintasa, } \\
\text { transportadores, permeasas y } \\
\text { enzimas catabólicas. }\end{array}$ & $\begin{array}{l}\text { Limitación de } \\
\text { nitrógeno }\end{array}$ & $\begin{array}{l}\text { (Ninfa, Atkinson, } \\
\text { Kamberov, \& Ninfa, } \\
\text { 1993) }\end{array}$ \\
\hline AtoS & E. coli & trans & Canónico & $\begin{array}{l}\text { Metabolismo de ácidos } \\
\text { grasos de cadena corta, } \\
\text { síntesis flagelar y } \\
\text { quimiotaxis }\end{array}$ & Acetoacetato & $\begin{array}{l}\text { (Filippou, Kasemian, } \\
\text { Panagiotidis, \& } \\
\text { Kyriakidis, 2008) }\end{array}$ \\
\hline AgrC & S. aureus & trans & Canónico & $\begin{array}{l}\text { Secreción de factores de } \\
\text { virulencia }\end{array}$ & $\begin{array}{l}\text { Ligando } \\
\text { autoinductor }\end{array}$ & $\begin{array}{l}\text { (George Cisar, } \\
\text { Geisinger, Muir, \& } \\
\text { Novick, 2009) }\end{array}$ \\
\hline VirA & $\begin{array}{l}\text { A. } \\
\text { tumefaciens }\end{array}$ & trans & Híbrido & $\begin{array}{l}\text { Genes del regulón vir } \\
\text { involucrados en la } \\
\text { transferencia de T-DNA }\end{array}$ & $\begin{array}{l}\text { Compuestos } \\
\text { fenólicos, acidez, } \\
\text { monosacáridos }\end{array}$ & $\begin{array}{l}\text { (Brencic, Xia, \& } \\
\text { Winans, 2004) }\end{array}$ \\
\hline RstB & E. coli & trans & Canónico & $\begin{array}{l}\text { Genes involucrados en } \\
\text { la tolerancia al ácido, } \\
\text { formación de fimbria y } \\
\text { respiración anaeróbica. }\end{array}$ & pH ácido & $\begin{array}{l}\text { (Ashenberg, Keating, \& } \\
\text { Laub, 2013) }\end{array}$ \\
\hline $\operatorname{Kin} \mathrm{A}$ & B. subtilis & trans & Canónico & $\begin{array}{l}\text { Regulación de la } \\
\text { esporulación. }\end{array}$ & $\begin{array}{l}\text { Pocos nutrientes, } \\
\text { señal desconocida }\end{array}$ & $\begin{array}{l}\text { (Devi, Kiehler, Haggett, } \\
\text { \& Fujita, 2015) }\end{array}$ \\
\hline DesK & B. subtilis & trans & Canónico & $\begin{array}{l}\text { Gen des, generación de } \\
\text { insaturaciones en ácidos } \\
\text { grasos. }\end{array}$ & $\begin{array}{l}\text { Cambios en la } \\
\text { fluidez de la } \\
\text { membrana por } \\
\text { temperatura }\end{array}$ & $\begin{array}{l}\text { (Trajtenberg, Graña, } \\
\text { Ruétalo, Botti, \& } \\
\text { Buschiazzo, 2010) }\end{array}$ \\
\hline $\mathrm{BvgS}$ & B. pertussis & trans & Híbrido & $\begin{array}{l}\text { Toxina pertussis, adenilato } \\
\text { ciclasa, fimbria y biogénesis } \\
\text { del flagelo. }\end{array}$ & $\begin{array}{l}\text { Señales } \\
\text { desconocidas }\end{array}$ & (Cotter \& Jones, 2003) \\
\hline EvgS & E. coli & cis & Híbrido & $\begin{array}{l}\text { Genes para la resistencia a } \\
\text { pH ácido. }\end{array}$ & $\begin{array}{l}\mathrm{pH} 5-6, \text { altas } \\
\text { concentraciones } \\
\text { de metales } \\
\text { alcalinos. }\end{array}$ & $\begin{array}{l}\text { (Kinoshita-Kikuta, } \\
\text { Kinoshita, Eguchi, \& } \\
\text { Koike, 2016) }\end{array}$ \\
\hline BarA & E. coli & trans & Híbrido & $\operatorname{csr} B$ y $\operatorname{csr} C$ & $\begin{array}{l}\text { Formato, acetato, } \\
\text { propionato }\end{array}$ & $\begin{array}{l}\text { (Kinoshita-Kikuta, } \\
\text { Kinoshita, Eguchi, \& } \\
\text { Koike, 2016) }\end{array}$ \\
\hline
\end{tabular}

Tabla I. Características de algunas cinasas sensoras bacterianas. 
CSs canónicas es catalizada por residuos conservados como Gln, Asn o Thr en el dominio transmisor, en un mecanismo muy semejante al utilizado por fosfatasas auxiliares como CheZ y CheX (Huynh, Noriega, \& Stewart, 2010). Además de los residuos catalíticos propuestos, la actividad fosfatasa del transmisor también requiere la correcta conformación de éste, así como de las interacciones apropiadas con el dominio receptor del RR. De esta manera, las conformaciones de cinasa y de fosfatasa de una CS deberían ser diferentes y mutuamente excluyentes, existiendo ambas conformaciones en un momento dado en equilibrio dinámico (Huynh \& Stewart, 2011). Así, el desplazamiento de este equilibrio hacia una u otra conformación, en respuesta a la presencia o ausencia de la señal específica, determina la relación entre un RR y su forma fosforilada y, por consiguiente, la magnitud de la respuesta fisiológica.

En el caso de las CSs híbridas, el mecanismo de desfosforilación del RR es sustancialmente diferente. En los sistemas BvgSBvgA de Bordetella pertussis y ArcB-ArcA de E. coli, se ha comprobado tanto in vivo como in vitro que el RR-P es desfosforilado mediante un fosforelevo reverso, que involucra la transferencia del $\sim$ P del Asp del RR a la His del HPt, luego al residuo de Asp del DR de la CS híbrida, y finalmente la liberación de fosfato inorgánico (Georgellis, Kwon, De Wulf, \& Lin, 1998; Peña-Sandoval, Kwon, \& Georgellis, 2005; Uhl $\&$ Miller, 1996a). Esto implica que los mismos residuos de Asp (en el DR) e His (en el HPt) de una CS híbrida están involucrados tanto en el fosforelevo como en el fosforelevo reverso, y que la transferencia del grupo $\sim \mathrm{P}$ entre el domino DR y el HPt es un proceso reversible. Entonces surgen nuevas interrogantes, como ¿qué determina la dirección de esta reacción? y, el modo de transferencia del grupo $\sim$ P, entre- o intra- monómeros, ¿se conserva en ambos sentidos?

\section{FOSFORELEVO Y FOSFORELEVO REVERSO EN LA CS HÍBRIDA ARCB}

El sistema $\mathrm{ArcB} / \mathrm{ArcA}$ de E. coli regula la expresión de una gran cantidad de genes en respuesta al estado redox del medio. Este SDC se encuentra activo en condiciones anaerobias y microaerobias de crecimiento, mientras que se inactiva cuando las células crecen en condiciones aerobias (Álvarez \& Georgellis, 2010; Georgellis, Kwon, \& Lin, 2001; Lynch \& Lin, 1996; Malpica, Sandoval, Rodríguez, Franco, \& Georgellis, 2006). ArcB es una cinasa híbrida y ha sido un modelo de estudio para este tipo de sensores, ya que fue uno de los pocos en los que se demostró que existe un fosforelevo y un fosforelevo reverso tanto in vivo como in vitro (Georgellis, Kwon, De Wulf, \& Lin, 1998; Georgellis, Lynch, \& Lin, 1997; Kwon, Georgellis, \& Lin, 2000; Peña-Sandoval, Kwon, \& Georgellis, 2005). El estudio del modo de autofosforilación y del de fosfotransferencia en $\mathrm{ArcB}$ ha sido abordado recientemente por distintos grupos de investigación y aplicando diferentes estrategias experimentales. Como se comentó anteriormente, se comprobó que ArcB se autofosforila en una reacción intramolecular (Kinoshita-Kikuta, Kinoshita, Eguchi, \& Koike, 2016; Peña-Sandoval \& Georgellis, 2010), en contra del paradigma instituido hasta ese momento. Pero fue en los pasos del fosforelevo en donde diferentes reportes llegaron a conclusiones disímiles. En un primer reporte, se hicieron ensayos in vivo de complementación de mutantes de ArcB en el fosforelevo (reemplazos puntuales en His292, Asp576 o His717), analizando los resultados con modelos matemáticos y estadísticos. En ningún caso lograron complementar la actividad del sistema al co-expresar dos formas mutantes de ArcB. Incluso, observaron que la co-expresión de una copia mutante en His292, Asp576 o His717 con ArcB silvestre, resultaba en un fenotipo comparable con una cepa mutante en $\operatorname{arc} B$, a pesar de expresarse la copia silvestre. Con estos resultados, los autores concluyeron que las fosfotransferencias en ArcB ocurrirían por un mecanismo cooperativo o alostérico, en el que todos los sitios fosforilables de un dímero de ArcB deben ser funcionales para que ocurra el fosforelevo (Jovanovic et al., 2015), ya que ni transferencias en trans ni en cis del grupo $\sim$ P explicaban los resultados obtenidos. En contraste, en un trabajo posterior en el que se analizó la capacidad de complementación de mutantes del fosforelevo de $A r c B$ in vitro, se sugirió que las dos transferencias de fosfato ocurren de manera intermolecular (trans) (KinoshitaKikuta, Kinoshita, Eguchi, \& Koike, 2016). En este caso, la fosforilación de los péptidos purificados se observó mediante el retardo en la migración de las formas fosforiladas en geles de poliacrilamida con un componente comercial denominado Phos-tag (Wako Pure Chemical Industries, Osaka, Japan). Ya que este método tiene una sensibilidad y reproducibilidad limitada, y requiere del uso de grandes concentraciones de proteína, los resultados reportados no fueron concluyentes. Finalmente, en un trabajo reciente de nuestro grupo, se abordó el estudio de los pasos del fosforelevo en ArcB, incluyendo, además, el paso del domino HPt al DR durante la desfosforilación de ArcA (fosforelevo reverso). En este caso se evaluó la capacidad de completar un fosforelevo funcional in vivo, de distintas mutantes en el fosforelevo de ArcB (simples y múltiples) expresadas y co-expresadas a niveles silvestres, a fin de evitar efectos fisiológicos indeseables producto de una sobreexpresión del sensor. También se analizó la complementación de mutantes in vitro, evaluando la fosforilación de la proteína ArcA (destino final del grupo $\sim \mathrm{P}$ en el fosforelevo), o la desfosforilación de ArcA-P, mediante el uso y detección de ${ }^{32} \mathrm{P}$. Los resultados de ambas estrategias experimentales comprobaron que la transferencia del grupo $\sim$ P desde el DT al DR es intramolecular (cis), y del DR al HPt es intermolecular (trans), mientras que el paso reverso desde el HPt al DR de ArcB es una fosfotransferencia intramolecular (cis) (Figura 3). Esto sugiere que la conformación de los dímeros de ArcB al actuar como cinasa aproxima los dominios DR y HPt de monómeros adyacentes (transferencia en trans), mientras que la estructura de dímeros de ArcB en 


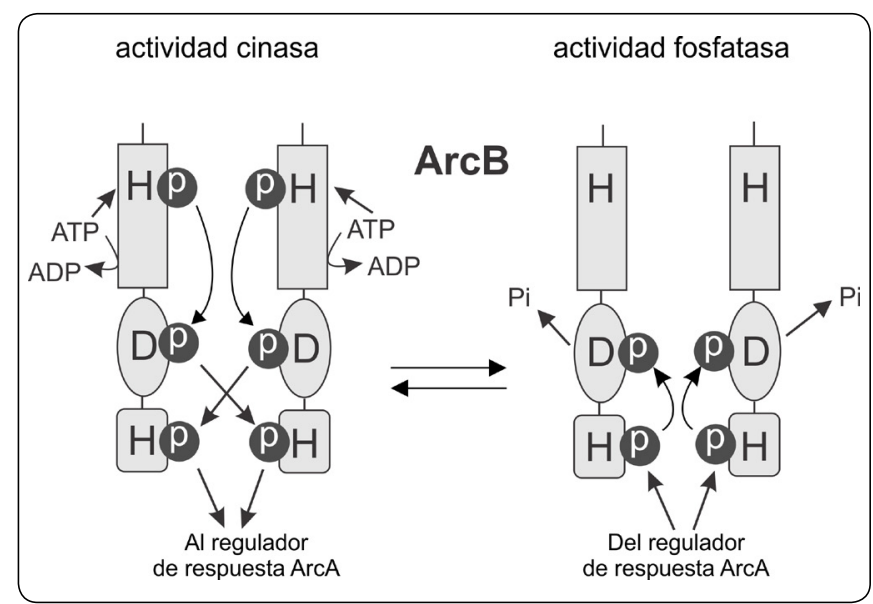

Figura 3. Modos de autofosforilación y transferencias del grupo fosforilo en la CS híbrida ArcB durante la activación e inactivación del sistema. Cuando las condiciones son estimulantes, ArcB actúa como cinasa y se autofosforila mediante una reacción intramolecular (cis). Luego transfiere el grupo $\sim P$ desde el DT al DR en cis, y del DR al HPt en trans. El paso reverso a esta última transferencia, cuando ArcB funciona como fosfatasa de ArcA, ocurre en cis. Fuente: elaboración propia.

estado de fosfatasa (en ausencia de la señal) empareja los dominios DR y HPt de un mismo monómero (transferencia en cis) (Figura 3). A su vez, podría imaginarse que estas conformaciones, mutuamente excluyentes y probablemente en un equilibrio dinámico, favorecidas por la presencia o la ausencia de la señal, determinan la dirección del grupo fosforilo hacia ArcA o hacia la liberación como fosfato inorgánico. Podría esperarse que otras CSs híbridas operasen de manera similar, dirigiendo el grupo $\sim \mathrm{P}$ hacia uno $\mathrm{u}$ otro destino de acuerdo con conformaciones estructurales que determinen fosfotransferencias intra o intermoleculares. Ya que no existen evidencias del modo de transferencia del grupo $\sim \mathrm{P}$ en el fosforelevo reverso para otra CS híbrida, lo anterior queda hasta el momento como una especulación.

\section{Conclusiones}

Los sistemas de dos componentes, basados en fosforilaciones y transferencias de grupos fosforilo entre residuos de His y Asp, son la principal vía de comunicación de las células bacterianas con su entorno. Desde la detección de una señal específica hasta la generación de una respuesta fisiológica, ocurren una serie de eventos que aseguran la eficiencia y fidelidad en la transducción de la señal. La transferencia de grupos $\sim P$ entre dominios de un SDC es parte de un mecanismo común para todos estos sistemas, y sin embargo, exhibe diferencias importantes entre sistemas particulares. El conocer la naturaleza de las transferencias de $\sim \mathrm{P}$ entre módulos de una CS híbrida brinda la oportunidad de predecir características estructurales de estas proteínas, así como diferencias entre las formas activas e inactivas de las mismas, que determinan la dirección del $\sim \mathrm{Py}$, en última instancia, modulan la respuesta adaptativa.

\section{Agradecimientos}

Los autores agradecen a la Dirección General de Asuntos del Personal Académico (DGAPA) de la UNAM, por el apoyo financiero a los proyectos IN209918 y IN208718. Juan Luis Terán Melo agradece al CONACYT por la beca de doctorado otorgada.

\section{REFERENCIAS}

Aguilar, P.S., Hernández-Arriaga, A. M., Cybulski, L. E., Erazo, A. C., \& de Mendoza, D. (2001). Molecular basis of thermosensing: a two-component signal transduction thermometer in Bacillus subtilis. The EMBO Journal, 20(7), 1681-1691. https://doi.org/10.1093/ emboj/20.7.1681

Álvarez, A. F., Barba-Ostria, C., Silva-Jiménez, H., \& Georgellis, D. (2016). Organization and mode of action of two component system signaling circuits from the various kingdoms of life. Environmental Microbiology, 18(10), 3210-3226. https://doi.org/10.1111/1462-2920.13397

Álvarez, A. F., \& Georgellis, D. (2010). In vitro and in vivo analysis of the $\mathrm{ArcB} / \mathrm{A}$ redox signaling pathway. Methods in Enzymology, 471(10), 205-228. https://doi. org/10.1016/S0076-6879(10)71012-0

Ashby, M. K. (2006). Distribution, structure and diversity of "bacterial" genes encoding two-component proteins in the Euryarchaeota. Archaea (Vancouver, B.C.), 2(1), 11-30. https://doi.org/10.1155/2006/562404

Ashenberg, O., Keating, A. E., \& Laub, M. T. (2013). Helix bundle loops determine whether histidine kinases autophosphorylate in cis or in trans. Journal of Molecular Biology, 425(7), 1198-1209. https://doi.org/10.1016/j. jmb.2013.01.011

Bourret, R. B., Hess, J. F., Borkovich, K. A., Pakula, A. A., \& Simon, M. I. (1989). Protein phosphorylation in chemotaxis and two-component regulatory systems of bacteria. The Journal of Biological Chemistry, 264(13), 7085-7088. Retrieved from http://www.ncbi.nlm.nih. gov/pubmed $/ 2540171$

Brencic, A., Xia, Q., \& Winans, S. C. (2004). VirA of Agrobacterium tumefaciens is an intradimer transphosphorylase and can actively block vir gene expression in the absence of phenolic signals. Molecular Microbiology, 52(5), 1349-1362. https://doi.org/10.1111/ j.1365-2958.2004.04057.x

Burbulys, D., Trach, K. A., \& Hoch, J. A. (1991). Initiation of sporulation in $B$. subtilis is controlled by a multicomponent phosphorelay. Cell, 64(3), 545-552. Retrieved from http://www.ncbi.nlm.nih.gov/pubmed/1846779

Casino, P., Rubio, V., \& Marina, A. (2009). Structural insight into partner specificity and phosphoryl transfer in twocomponent signal transduction. Cell, 139(2), 325-336. https://doi.org/10.1016/j.cell.2009.08.032

Catlett, N. L., Yoder, O. C., \& Turgeon, B. G. (2003). Wholegenome analysis of two-component signal transduction 
genes in fungal pathogens. Eukaryotic Cell, 2(6), 11511161. https://doi.org/10.1128/EC.2.6.1151-1161.2003

Cotter, P. A., \& Jones, A. M. (2003). Phosphorelay control of virulence gene expression in Bordetella. Trends in Microbiology, 11(8), 367-373. https://doi.org/10.1016/ S0966-842X(03)00156-2

Devi, S. N., Kiehler, B., Haggett, L., \& Fujita, M. (2015). Evidence that autophosphorylation of the major sporulation kinase in Bacillus subtilis is able to occur in trans. Journal of Bacteriology, 197(16), 2675-2684. https://doi.org/10.1128/JB.00257-15

Dutta, R., Qin, L., \& Inouye, M. (1999). Histidine kinases: diversity of domain organization. Molecular Microbiology, 34(4), 633-640. https://doi.org/10.1046/ j.1365-2958.1999.01646.x

Eguchi, Y., \& Utsumi, R. (2014). Alkali metals in addition to acidic $\mathrm{pH}$ activate the EvgS histidine kinase sensor in Escherichia coli. Journal of Bacteriology, 196(17), 3140-3149. https://doi.org/10.1128/JB.01742-14

Filippou, P. S., Kasemian, L. D., Panagiotidis, C. A., \& Kyriakidis, D. A. (2008). Functional characterization of the histidine kinase of the E. coli two-component signal transduction system AtoS-AtoC. Biochimica et Biophysica Acta, 1780(9), 1023-1031. https://doi. org/10.1016/j.bbagen.2008.05.002

Forst, S. A., \& Roberts, D. L. (1994). Signal transduction by the EnvZ-OmpR phosphotransfer system in bacteria. Research in Microbiology, 145(5-6), 363-373. https:// doi.org/10.1016/0923-2508(94)90083-3

Galperin, M. Y. (2005). A census of membrane-bound and intracellular signal transduction proteins in bacteria: bacterial IQ, extroverts and introverts. BMC Microbiology, 5, 35. https://doi.org/10.1186/1471-2180-5-35

Gao, R., \& Stock, A. M. (2009). Biological insights from structures of two-component proteins. Annual Review of Microbiology, 63, 133-154. https://doi.org/10.1146/ annurev.micro.091208.073214

George Cisar, E. A., Geisinger, E., Muir, T. W., \& Novick, R. P. (2009). Symmetric signalling within asymmetric dimers of the Staphylococcus aureus receptor histidine kinase AgrC. Molecular Microbiology, 74(1), 44-57. https://doi.org/10.1111/j.1365-2958.2009.06849.x

Georgellis, D., Kwon, O., De Wulf, P., \& Lin, E. C. (1998). Signal decay through a reverse phosphorelay in the Arc two-component signal transduction system. J. Biol. Chem., 273(49), 32864-32869. https://doi.org/10.1074/ jbc.273.49.32864

Georgellis, D., Kwon, O., \& Lin, E. C. (2001). Quinones as the redox signal for the Arc two-component system of bacteria. Science (New York, N.Y.), 292(5525), 23142316. https://doi.org/10.1126/science.1059361

Georgellis, D., Lynch, A. S., \& Lin, E. C. (1997). In vitro phosphorylation study of the Arc two-component signal transduction system of Escherichia coli. J.
Bacteriol., 179(17), 5429-5435. https://doi.org/10.1128/ jb.179.17.5429-5435.1997

Hutchings, M. I., Hong, H.-J., \& Buttner, M. J. (2006). The vancomycin resistance VanRS two-component signal transduction system of Streptomyces coelicolor. Molecular Microbiology, 59(3), 923-935. https://doi. org/10.1111/j.1365-2958.2005.04953.x

Huynh, T. N., Noriega, C. E., \& Stewart, V. (2010). Conserved mechanism for sensor phosphatase control of twocomponent signaling revealed in the nitrate sensor NarX. Proc. Natl. Acad. Sci. U S A, 107(49), 21140-21145. https://doi.org/10.1073/pnas.1013081107

Huynh, T. N., \& Stewart, V. (2011). Negative control in twocomponent signal transduction by transmitter phosphatase activity. Molecular Microbiology, 82(2), 275-286. https:// doi.org/10.1111/j.1365-2958.2011.07829.x

Iuchi, S., \& Lin, E. C. C. (1993). Adaptation of Escherichia coli to redox environments by gene expression. Molecular Microbiology, 9(1), 9-15. https://doi. org/10.1111/j.1365-2958.1993.tb01664.x

Jiang, M., Shao, W., Perego, M., \& Hoch, J. A. (2000). Multiple histidine kinases regulate entry into stationary phase and sporulation in Bacillus subtilis. Molecular Microbiology, 38(3), 535-542. https://doi.org/10.1046/ j.1365-2958.2000.02148.x

Jourlin, C., Ansaldi, M., \& Mejean, V. (1997). Transphosphorylation of the TorR response regulator requires the three phosphorylation sites of the TorS unorthodox sensor in Escherichia coli. Journal of Molecular Biology, 267(4), 770-777. https://doi. org/10.1006/jmbi.1997.0919

Jovanovic, G., Sheng, X., Ale, A., Feliu, E., Harrington, H. A., Kirk, P., Wiuf, C., Buck, M., \& Stumpf, M. P. H. (2015). Phosphorelay of non-orthodox two component systems functions through a bi-molecular mechanism in vivo: the case of ArcB. Mol. BioSyst. Mol. BioSyst, 11(11), 13481359. https://doi.org/10.1039/c4mb00720d

Kenney, L. J. (2010). How important is the phosphatase activity of sensor kinases? Current Opinion in Microbiology, 13(2), 168-176. https://doi.org/10.1016/j. mib.2010.01.013

Kinoshita-Kikuta, E., Kinoshita, E., Eguchi, Y., \& Koike, T. (2016). Validation of cis and trans modes in multistep phosphotransfer signaling of bacterial tripartite sensor kinases by using Phos-Tag SDS-PAGE. PLOS ONE, 11(2), e0148294. https://doi.org/10.1371/journal.pone.0148294

Koretke, K. K., Lupas, A. N., Warren, P. V, Rosenberg, M., \& Brown, J. R. (2000). Evolution of two-component signal transduction. Molecular Biology and Evolution, 17(12), 1956-1970. https://doi.org/doi.org/10.1093/ oxfordjournals.molbev.a026297

Kwon, O., Georgellis, D., \& Lin, E. C. (2000). Phosphorelay as the sole physiological route of signal transmission by the Arc two-component system of Escherichia coli. J. 
Bacteriol., 182(13), 3858-3862. https://doi.org/10.1128/ JB.182.13.3858-3862.2000

Levit, M., Liu, Y., Surette, M., \& Stock, J. (1996). Active Site Interference and Asymmetric Activation in the Chemotaxis Protein Histidine Kinase CheA. Journal of Biological Chemistry, 271(50), 32057-32063. https://doi. org/10.1074/jbc.271.50.32057

Lynch, A. S., \& Lin, E. C. (1996). Regulation of gene expression in Escherichia coli. In F. C. Neidhardt, R. Curtis, A. L. Ingraham, E. C. C. Lin, K. B. Low, B. Magasanik, W. S. Reznikoff, M. Riley, M. Schaechter, \& H. E. Umbarger (Eds.), Escherichia coli and Salmonella: Cellular and Molecular Biology (pp. 1526-1538). Washington, DC: Am. Soc. Microbiol.

Malpica, R., Sandoval, G. R., Rodriguez, C., Franco, B., \& Georgellis, D. (2006). Signaling by the Arc twocomponent system provides a link between the redox state of the quinone pool and gene expression. Antioxid. Redox Signal, 8(5-6), 781-795. https://doi.org/10.1089/ ars.2006.8.781

Ninfa, E. G., Atkinson, M. R., Kamberov, E. S., \& Ninfa, A. J. (1993). Mechanism of autophosphorylation of Escherichia coli nitrogen regulator II (NRII or NtrB): trans-phosphorylation between subunits. Journal of Bacteriology, 175(21), 7024-7032. https://doi. org/10.1128/jb.175.21.7024-7032.1993

Nohno, T., Noji, S., Taniguchi, S., \& Saito, T. (1989). The narX and narL genes encoding the nitrate-sensing regulators of Escherichia coli are homologous to a family of prokaryotic two-component regulatory genes. Nucleic Acids Research, 17(8), 2947-2957. Retrieved from http:// www.ncbi.nlm.nih.gov/pubmed/2657652

Peña-Sandoval, G. R., \& Georgellis, D. (2010). The ArcB sensor kinase of Escherichia coli autophosphorylates by an intramolecular reaction. Journal of Bacteriology, 192(6), 1735-1739. https://doi.org/10.1128/JB.0140109

Peña-Sandoval, G. R., Kwon, O., \& Georgellis, D. (2005). Requirement of the receiver and phosphotransfer domains of ArcB for efficient dephosphorylation of phosphorylated ArcA in vivo. Journal of Bacteriology, 187(9), 32673272. https://doi.org/10.1128/JB.187.9.3267-3272.2005

Santos, J. L., \& Shiozaki, K. (2001). Fungal histidine kinases. Science's STKE, 2001(98), re1. https://doi.org/10.1126/ stke.2001.98.re1

Schaller, G. E., Shiu, S.-H., \& Armitage, J. P. (2011). Twocomponent systems and their co-option for eukaryotic signal transduction. Current Biology : $C B, 21(9)$, R320330. https://doi.org/10.1016/j.cub.2011.02.045

Stock, A. M., Robinson, V. L., \& Goudreau, P. N. (2000). Two-component signal transduction. Annual Review of Biochemistry, 69, 183-215. https://doi.org/10.1146/ annurev.biochem.69.1.183

Swanson, R. V, Bourret, R. B., \& Simon, M. I. (1993).
Intermolecular complementation of the kinase activity of CheA. Molecular Microbiology, 8(3), 435-441. https:// doi.org/10.1111/j.1365-2958.1993.tb01588.x

Takeda, S., Fujisawa, Y., Matsubara, M., Aiba, H., \& Mizuno, T. (2001). A novel feature of the multistep phosphorelay in Escherichia coli: a revised model of the RcsC --> YojN --> RcsB signalling pathway implicated in capsular synthesis and swarming behaviour. Molecular Microbiology, 40(2), 440-450. https://doi.org/10.1046/ j.1365-2958.2001.02393.x

Tanaka, T., Saha, S. K., Tomomori, C., Ishima, R., Liu, D., Tong, K. I., Park, H., Dutta, R., Qing, L., Swindells, M. B., Yamazaki, T., Ono, A. M., Kainosho, M., Inouye, M., \& Ikura, M. (1998). NMR structure of the histidine kinase domain of the E. coli osmosensor EnvZ. Nature, 396(6706), 88-92. https://doi.org/10.1038/23968

Terán-Melo, J. L., Peña-Sandoval, G. R., Silva-Jiménez, H., Rodríguez, C., Álvarez, A. F., \& Georgellis, D. (2018). Routes of phosphoryl group transfer during signal transmission and signal decay in the dimeric sensor histidine kinase ArcB. The Journal of Biological Chemistry, 293(34), 13214-13223. https://doi. org/10.1074/jbc.RA118.003910

Thomason, P., \& Kay, R. (2000). Eukaryotic signal transduction via histidine-aspartate phosphorelay. Journal of Cell Science, 113, 3141-3150.

Trajtenberg, F., Graña, M., Ruétalo, N., Botti, H., \& Buschiazzo, A. (2010). Structural and enzymatic insights into the ATP binding and autophosphorylation mechanism of a sensor histidine kinase. The Journal of Biological Chemistry, 285(32), 24892-24903. https://doi. org/10.1074/jbc.M110.147843

Uhl, M. A., \& Miller, J. F. (1996a). Central role of the BvgS receiver as a phosphorylated intermediate in a complex two-component phosphorelay. The Journal of Biological Chemistry, 271(52), 33176-33180. https://doi. org/10.1074/jbc.271.52.33176

Uhl, M. A., \& Miller, J. F. (1996b). Integration of multiple domains in a two-component sensor protein: the Bordetella pertussis BvgAS phosphorelay. The EMBOJournal, 15(5), 1028-1036. https://doi.org/10.1002/j.1460-2075.1996. tb00440.x

Ulrich, L. E., \& Zhulin, I. B. (2010). The MiST2 database: a comprehensive genomics resource on microbial signal transduction. Nucleic Acids Research, 38(Database issue), D401-407. https://doi.org/10.1093/nar/gkp940

West, A. H., \& Stock, A. M. (2001). Histidine kinases and response regulator proteins in two-component signaling systems. Trends in Biochemical Sciences, 26(6), 369376. https://doi.org/10.1016/S0968-0004(01)01852-7

Williams, R. H. N., \& Whitworth, D. E. (2010). The genetic organisation of prokaryotic two-component system signalling pathways. BMC Genomics, 11, 720. https://doi. org/10.1186/1471-2164-11-720 
Wolanin, P. M., Thomason, P. A., \& Stock, J. B. (2002). Histidine protein kinases: key signal transducers outside the animal kingdom. Genome Biology, 3(10), 1-8. https:// doi.org/10.1186/gb-2002-3-10-reviews3013

Wuichet, K., Cantwell, B. J., \& Zhulin, I. B. (2010). Evolution and phyletic distribution of two-component signal transduction systems. Current Opinion in
Microbiology, 13(2), 219-225. https://doi.org/10.1016/j. mib.2009.12.011

Yang, Y., \& Inouye, M. (1991). Intermolecular complementation between two defective mutant signaltransducing receptors of Escherichia coli. Proc. Natl. Acad. Sci. U S A, 88(24), 11057-11061. https://doi. org/10.1073/pnas.88.24.11057 